LBL - 35929

\title{
Properties of Strange-Matter Stars ${ }^{1}$
}

\author{
Fridolin Weber and Christiane Kettner \\ Institute of Theoretical Physics, University of Munich, Theresienstr. 97/III, 80999 Munich, \\ Germany \\ and \\ Norman K. Glendenning \\ Nuclear Science Division, Lawrence Berkeley Laboratory, Berkeley, CA 94720, USA
}

July 27, 1994
Presented at the International Conference
Nuclei in the Cosmos
Gran Sasso, Italy
July 8-13, 1994

To be published in the Proceedings

${ }^{1}$ This work was supported by the Director, Office of Energy Research, Office of High Energy and Nuclear Physics, Division of Nuclear Physics, of the U.S. Department of Energy under Contract DE-AC03-76SF00098. 


\title{
Properties of Strange-Matter Stars
}

\author{
F. Weber(1), Ch. Kettner(1), and N. K. Glendenning(2) \\ (1) Institute of Theoretical Physics, University of Munich, Theresienstr. 97/1 ;0999 Munich, \\ Germany, and (2) Nuclear Science Division, Lawrence Berkeley Laboratory, $B_{\epsilon}$ :eley, CA 94720, \\ USA
}

\begin{abstract}
This paper deals with an investigation of the properties of hypothetical strange-matter stars, which are composed of $u, d, s$ quark matter whose energy per baryon number lies below the one of ${ }^{56} \mathrm{Fe}$ (Witten's strange matter hypothesis' $\mathrm{Ob}$ servable quantities which allow to distinguish such objects from their "convent. onal" counterparts, neutron stars and white dwarfs, are pointed out.
\end{abstract}

Key words: Strange Matter - Strange Stars - Pulsars

\section{INTRODUCTION}

The hypothesis that strange quark matter may be the absolute ground state of the strong interaction (not ${ }^{56} \mathrm{Fe}$ ) has been raised by Witten in 1984 (Witten 1984). If the hypothesis is true, then a separate class of compact stars could exist, which are called strange stars. They form a distinct and disconnected branch of compact stars and are not part of the continuum of equilibrium configurations that include white dwarfs and neutron stars. In principle both strange and neutron stars could exist. However if strange stars exist, the galaxy is likely to be contaminated by strange quark nuggets which would convert all neutron stars that they come into contact with to strange stars (Glendenning 1990, Madsen and Olesen 1991, Caldwell and Friedman 1991). This in turn means that the objects known to astronomers as pulsars are probably rotating strange matter stars, not neutron matter stars as is usually assumed. The properties of (rotating) strange stars are discussed in this paper. 


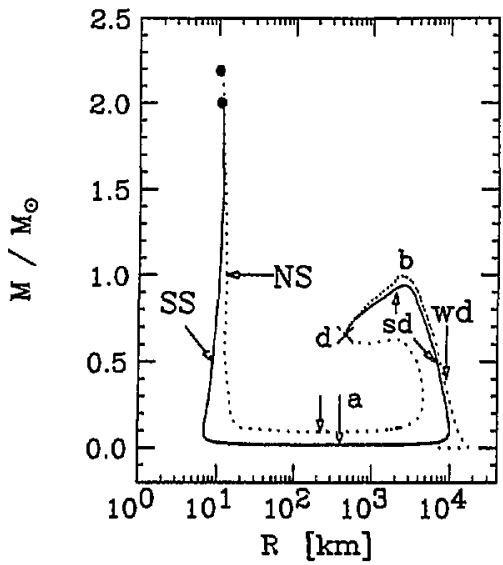

Fig. 1.- Mass versus radius of strange-star configurations with nuclear crust (solid curve) and gravitationally bound stars (dotted curve). The following abbreviations are used: $\mathrm{NS}=$ neutron star, $\mathrm{SS}=$ strange star, $w d=$ white dwarf, sd=strange dwarf.

\section{NEUTRON STARS AND WHITE DWARFS VERSUS STRANGE-MATTER STARS}

\subsection{Minimum Rotational Periods of Neutron Stars}

From our extensive investigation performed somewhere else (Weber and Glendenning 1993a), it is know that the gravitational-radjation reaction driveis instability sets a lower limit on the stable rotational period of a rotating neutron star of mass $\sim 1.45 M_{\odot}$ of about $1 \mathrm{msec}$. (For the purpose of comparison, the most rapidly rotating pulsar known to date rotates at $1.56 \mathrm{msec}$.) This indicates ihat the nature of any pulsar that is found to have a considerably shorter period than $\sim 1$ msec must be different from the one of a neutron star. As poinfed out in the literature, rapidly rotating strange stars arise as natural candidates for such objects (Glendenning 1990). Being bound by the strong force rather than gravity, strange stars possess a mass-radius relationship (cf. Sect. 3.) that allows them to withstand such rapid rotation.

\section{Mass-Radius Relationships}

The mass-radius relationship of non-rotating strange stars with nuclear crust, whose inner density is equal to neutron drip (maximum possible density, Alcock et al. 1986) is shown in Fig. 1 (Weber and Glendenning 1993b, Weber and Glendenning $1993 \mathrm{c}$ ). A value for the bag constant of $B^{1 / 4}=145 \mathrm{MeV}$ has been chosen. This choice represents strongly bound strange matter with an energy per baryon $\sim 830 \mathrm{MeV}$. The solid dots denote the maximum-mass stars of the neutron (NS) and strange quark star (SS) sequences. The ariows indicate the minimum-mass star of each sequence (' $a$ ': strange star, ' $b$ ': neutron star). White dwarf-like strange star configurations ('sd': strange dwarfs) terminate at the crossed point labeled ' $d$ '. The symbol 'wd' indicates the region of ordinary white dwarfs. The sequence of strange stars has a minimum mass of $\sim 0.015 M_{\odot}$ (radius of $\sim 400 \mathrm{~km}$ ) or about 15 Jupiter masses, which is smaller than that of the neutron star sequence, about $0.1 M_{\odot}$ (Baym et al. 


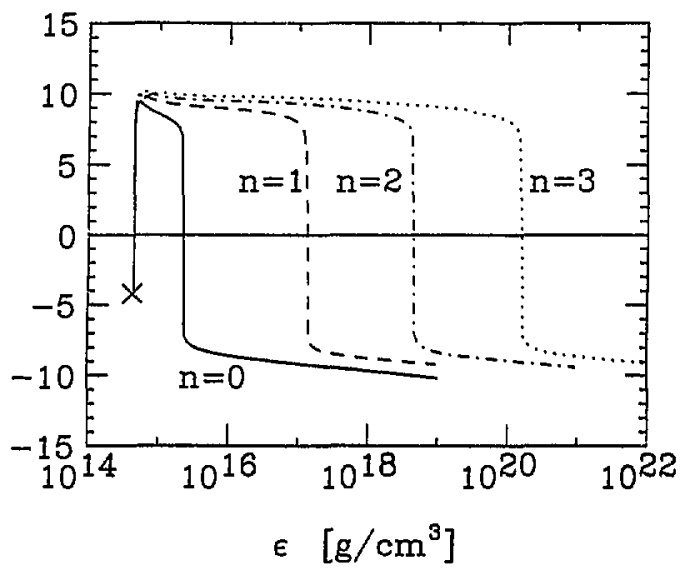

Fig. 2.- Pulsation frequencies, $\omega_{n}^{2}$, of the lowest four ( $n=0,1,2$, and 3 ) normal radial modes of strange stars as a function of central star density. Instead of $\omega_{n}^{2}$ itself, the quantity $\operatorname{sign}(a) \log (1+\operatorname{abs}(a))$, where $a \equiv\left(\omega_{n} / \sec ^{-1}\right)^{2}$, is plotted on the y-axis. The cross refers to the termination point of the strange dwarf sequence (cf. Fig. 1).

1971). These low-mass strange stars may be of considerable importance since they may be difficult to detect and therefore may effectively hide baryonic matter. It is striking that the bulk properties of neutron and strange stars of masses that are typical for neutron stars, $1.1 \lesssim M / M_{\odot} \lesssim 1.8$, are relatively similar and therefore do not allow the distinction between the two different species of stars. The situation changes as regards the possibility of fast rotation of strange stars. This has its origin in the different mass-radius relationships of neutron stars and strange quark stars (Glendenning 1990). As a consequence of this the entire family of strange stars can rotate rapidly, at Kepler periods in the range $\sim(0.4-0.8)$ msec (depending on mass and bag constant), not just those near the limit of gravitational collapse to a black hole as is the case for neutron stars (Glendenning and Weber 1992).

\section{STABILITY OF STRANGE-MATTER STARS AGAINST RADIAL OSCILLATIONS}

The normal modes of vibration of the strange-matter stars are computed from the Sturm-Liouville eigenequation, possessing the form (for details, see Glendenning et al. 1993),

$$
\frac{d}{d r}\left(\Pi(r) \frac{d u_{n}(r)}{d r}\right)+\left(Q(r)+\omega_{n}^{2} W(r)\right) u_{n}(r)=0 .
$$

The four lowest-lying eigenfrequencies of massive strange stars and strange dwarfs, whose inner crust density is equal to neutron drip, are shown in Fig. 2. We find that the $n=0$ mode becomes zero for the maxirnum- and minimum-mass star configurations labeled ' $a$ ' and ' $b$ ' in Fig. 1 (Glendenning et al. 1993). The $n=0$ mode passes through zero at ' $b$ ' and remains negative (i.e., $\omega_{n}^{2}<0$ ) for all densities down to the central density of the strange dwarf star at the termination point (cross). Since $\omega_{0}^{2}<0$ is associated with an exponentially growing mode of oscillation, all these strange dwarfs are unstable against radial oscillations. Thus, no stable strange $d w a r i s$ located between ' $b$ ' and the crossed termination point can exist in nature! 


\section{SUMMARY}

The main issues of this contribution can be stated as follows:

- Strange pulsars of masses $M \sim 1.45 M_{\odot}$ can have rotational periods that are clearly smaller than 1 millisecond. Because this range seems to be excluded for neutron stars, submillisecond pulsar periods may serve as signatures for strange stars.

- Strange stars can possess nuclear crusts of thickness $\sim\left(1-10^{3}\right) \mathrm{km}$, depending on central density. This will be of great importance for their cooling behavior.

- Strange stars possess masses in the range $\sim\left(2-10^{-4}\right) M_{\odot}$ and radii $\sim 10^{3}$ $\mathrm{km}$. Since masses and radii of $10^{-4} M_{\odot}$ and $\sim 10^{3} \mathrm{~km}$ are cornpletely excluded for both neutron stars as well as white dwarfs, they may serve as additional signatures for hypothetical strange stars.

- The Oppenheimer-Volkoff equations lead to strange-star configurations whose masses and radii are similar to those of ordinary white dwarfs. We thus call such objects strange dwarfs. Those strange dwarfs carrying nuclear crusts whose density at the base is equal to neutron drip are unstable against radial oscillations.

\section{REFERENCES}

Witten, E. 1984, Phys. Rev. D, 30, 272

Glendenning, N. K. 1990, Mod. Phys. Lett., A5, 2197

Madsen, J., and Olesen, M. L. 1991, Phys. Rev. D, 43, 1069; ibid., 44, 4150 (erratum)

Caldwell, R. R., and Friedman, J. L. 1991, Phys. Lett., 264B, 143

Weber, F., and Glendenning, N. K. 1993a, Astrophysics and Neutrino Physics, Eds. D. H. Feng, G. Z. He, and X. Q. Li, World Scientific, $63-183$

Alcock, C, Farhi, E., and Olinto, A. V. 1986, ApJ, 310, 261

Weber, F., and Glendenning, N. K. 1993b, Proceedings of the 2nd International Conference on Physics and Astrophysics of Quark-Gluon Plasma, January 19-23, 1993, Calcutta, India, to be published by World Scientific, (LBL-33771)

Weber, F., and Glendenning, N. K. 1993c, Proceedings of the NATO Advanced Study Institute Hot and Dense Nuclear Matter, Bodrum/Turkey, 26.09.-9.10.1993, ed. by W. Greiner and H. Stöcker, to be published by Plenum Press, (LBL-34783)

Bayn., G., Pethick, C, and Sutherland, P. 1971, ApJ, 170299

Glendenning, N. K., and Weber, F. 1992, ApJ, 400, 647

Glendenning, N. K., Kettner, Ch., and Weber, F. 1993, Properties of Strange Stars and Strange Dwarfs, in preparation, (LBL 34869) 